\title{
A toolset to study functions of Cytosolic non-specific dipeptidase 2 (CNDP2) using Drosophila as a model organism
}

\author{
Evgeniya N. Andreyeva ${ }^{1 *+}$, Anna A. Ogienko ${ }^{1,2+}$, Tatiana D. Dubatolova ${ }^{1}$, Anastasiya L. Oshchepkova ${ }^{1,3}$, \\ Elena N. Kozhevnikova', Anton V. Ivankin ${ }^{1}$, Gera A. Pavlova', Sergei A. Kopyl ${ }^{1}$ and Alexey V. Pindyurin ${ }^{1,2^{*}}$
}

From 11th International Multiconference "Bioinformatics of Genome Regulation and StructurelSystems Biology" - BGRSISB-

2018

Novosibirsk, Russia. 20-25 August 2018

\begin{abstract}
Background: Expression of the CNDP2 gene is frequently up- or down-regulated in different types of human cancers. However, how the product of this gene is involved in cell growth and proliferation is poorly understood. Moreover, our knowledge of the functions of the CNDP2 orthologs in well-established model organisms is scarce. In particular, the function of the D. melanogaster ortholog of CNDP2, encoded by the CG17337 gene (hereafter referred to as $d(N D P 2)$, is still unknown.

Results: This study was aimed at developing a set of genetic and molecular tools to study the roles of $d C N D P 2$. We generated a dCNDP2 null mutation (hereafter $\triangle d C N D P 2)$ using CRISPR/Cas9-mediated homologous recombination (HR) and found that the $\triangle d C N D P 2$ mutants are homozygous viable, morphologically normal and fertile. We also generated transgenic fly lines expressing eGFP-tagged and non-tagged dCNDP2 protein, all under the control of the UAS promoter, as well as polyclonal antibodies specific to dCNDP2. Using these tools, we demonstrate that only one of the two predicted dCNDP2 isoforms is expressed throughout the different tissues tested. dCNDP2 was detected in both the cytoplasm and the nucleus, and was found to be associated with multiple sites in the salivary gland polytene chromosomes.

Conclusions: The dCNDP2 gene is not essential for fly viability under standard laboratory conditions. The subcellular localization pattern of dCNDP2 suggests that this protein might have roles in both the cytoplasm and the nucleus. The genetic and molecular tools developed in this study will allow further functional characterization of the conserved CNDP2 protein using D. melanogaster as a model system.
\end{abstract}

Keywords: Drosophila melanogaster, CG17337, CNDP2, CN2, CPGL, CRISPR/Cas9, Tumor suppressor gene

\section{Background}

Peptidases with different substrate specificities play distinct roles in protein and peptide metabolism in eukaryotes. Mammalian CNDP2 (also known as carnosine dipeptidase II, CN2, carboxypeptidase of glutamate-like, CPGL) belongs to the M20 family of metallopeptidases

\footnotetext{
* Correspondence: andreeva@mcb.nsc.ru; a.pindyurin@mcb.nsc.ru

†Evgeniya N. Andreyeva and Anna A. Ogienko contributed equally to this work.

${ }^{1}$ Institute of Molecular and Cellular Biology, Siberian Branch of the Russian Academy of Sciences, Novosibirsk 630090, Russia

Full list of author information is available at the end of the article
}

and has broad substrate specificity for dipeptides $[1,2]$. It is active only as a homodimer [3], and the catalytic domain of each dimer subunit has one active center with two $\mathrm{Mn}^{2+}$ or $\mathrm{Zn}^{2+}$ ions, which determine the specificity of the enzyme for its physiological substrates [4]. So far, CNDP2 is the only known protease that can catalyze the formation of pseudodipeptides of lactic acid and amino acids ( $N$-lactoyl-amino acids) through reverse proteolysis in vivo [5]. Thus, besides its proteolytic activity, CNDP2 might perform other cellular functions.

(c) The Author(s). 2019 Open Access This article is distributed under the terms of the Creative Commons Attribution 4.0 International License (http://creativecommons.org/licenses/by/4.0/), which permits unrestricted use, distribution, and 
An aberrant expression of $C N D P 2$ is associated with tumorigenesis in humans. A decreased CNDP2 level was observed in pancreatic cancer, hepatocellular carcinoma and gastric cancer [2, 6, 7]. The CNDP2 isoform, CPGL-B, which lacks part of the catalytic domain, has been also implicated in tumor suppression, but it is currently unknown whether CPGL-B has peptidase activity $[2,6,7]$. However, not all tumors are characterized by low CNDP2 levels. An upregulated expression of CNDP2 has been indeed observed in breast carcinoma, and in kidney and colon cancers [8-11]. Several studies have been devoted to understanding the contribution of CNDP2 to carcinogenesis [2, 6-11], but an animal model with mutated $C N D P 2$ is not currently available.

The CNDP2 protein is highly conserved across species $[1,12-14]$ and ubiquitously expressed ([1]; A Database of Drosophila Genes \& Genomes available from flybase.org [15]). In mouse and human cells, CNDP2 localizes to the cytosol and the nucleoplasm (Human Protein Atlas available from www.proteinatlas.org [16]); in transiently transfected Chinese hamster ovary $(\mathrm{CHO})$ cells, CNDP2 was found in the cytoplasmic fraction [1]. In D. melanogaster, there is only one CNDP2 gene (CG17337; flybase.org [15]), hereafter referred to as $d C N D P 2$. Alignment of the amino acid sequences of the longest isoform of human CNDP2 (475 amino acids; GenPept accession no. NP_060705.2) and the longest isoform of Drosophila dCNDP2 (478 amino acids; GenPept accession no. NP_610181.2) revealed 63\% sequence identity along the entire length of the polypeptide. Among the few D. melanogaster genes encoding the M20 metallopeptidase proteins, only $d C N D P 2$ is ubiquitously expressed with moderate to high level (flybase.org [15]). The product of this gene was shown to be an extracellular component of larval hemolymph $[17,18]$, but its subcellular localization is unknown.

To address the role of CNDP2 in cell growth and proliferation we decided to exploit $D$. melanogaster as a model system. The ease of genomic manipulations in flies allows investigation of processes at both the organismal and cellular levels. In this study, we generated a null $d C N D P 2$ mutant, transgenic lines for inducible $d C N D P 2$ expression, polyclonal antibodies against dCNDP2 and use these tools to provide an initial characterization of the protein.

\section{Methods}

\section{Fly stocks}

Flies were raised and crossed at $25^{\circ} \mathrm{C}$ according to standard procedures. The following lines from the Bloomington Drosophila Stock Center (Bloomington, IN; bdsc.indiana.edu) were used: \#1824 $\left(y^{1} w^{*} ; P\left\{w^{+m W \cdot h s}=G a w B\right\} A B 1\right)$; \#32186 ( $w^{*} ; \quad P\left\{y^{+t 7.7} w^{+m C}=10 x U A S-I V S-m C D 8:: G F-\right.$ P\}attP40); \#4775 ( $\left.w^{1118} ; P\left\{w^{+m C}=U A S-G F P . n l s\right\} 14\right) ; \# 24488$ $\left(y^{1} M\{\right.$ vas-int.Dm $\left.\} Z H-2 A \quad w^{*} ; M\{3 x P 3-R F P . a t t P\} Z H-102 D\right)$;
\#51325 $\quad\left(w^{1118} ; \quad\right.$ PBac $\left\{y^{+m D i n t 2}=\right.$ vas-Cas9,U6-tracrRNA\}VK00027) and \#6599 $\left(y^{1} w^{67 c 23}\right)$ as a wild-type control. The stock carrying the nanos-Cre transgene [19] and the $P\{$ CaryP\}attP154 line [20] were kindly provided by Stepan N. Belyakin and Sergei A. Demakov, respectively (IMCB SB RAS, Novosibirsk, Russia).

\section{Plasmid constructs}

Twenty-nucleotide guide RNA (gRNA) sequences for the $d C N D P 2$ gene, gRNA1 (5'-GUAAAAUAGAUUCG ACGUAA-3') and gRNA2 (5'-GAACCAGAUAUGAC CCGCGA-3'), were designed using the CRISPR Design tool (zlab.bio/guide-design-resources). Their corresponding DNA sequences were cloned into the pU6-BbsI-chiRNA plasmid vector [21] downstream of the Drosophila U6 promoter by using the BbsI restriction sites to produce pU6-5'dCNDP2-chiRNA and pU6-3'dCNDP2-chiRNA constructs. Each plasmid expresses chimeric RNA (chiRNA) composed of the tracrRNA from Streptococcus pyogenes with the specific 20-nt gRNA sequence at the $5^{\prime}$ end [21]. The plasmid vector pU6-BbsI-chiRNA was a gift from Melissa Harrison \& Kate O'Connor-Giles \& Jill Wildonger (Addgene plasmid \#45946).

The plasmid construct pGX-5'\&3'-dCNDP2-null containing a "GMR enhancer-mini-white gene" reporter cassette placed between two DNA fragments, which flank the $d C N D P 2$ gene in the D. melanogaster genome and here referred to as $5^{\prime}$ - and $3^{\prime}$-homology arms (or simply the left and right arms), was made as follows. First, the left arm (2R:5,695,002-5,696,806; here and afterwards, coordinates are from Release 6 of the D. melanogaster genome assembly [22]) was cloned into the pGX-attP plasmid vector [23] using the unique NotI and KpnI sites. This yielded the intermediate pGX-5' -dCNDP2-null plasmid. Next, the right arm (2R:5,701,122-5,703,566) was cloned into the pGX-5' $-\mathrm{dCNDP} 2$-null plasmid by using the unique AscI and XhoI sites to produce the pGX-5' \& $3^{\prime}-\mathrm{dCNDP} 2$-null construct. The cloned right arm contains the following three single nucleotide variations, all upstream of the $v l c$ gene transcription start site: 5,701,650 $\mathrm{T}>\mathrm{C}, 5,701,704$ $5,701,705 i n s A$ and 5,701,862 $\mathrm{T}>\mathrm{C}$. The plasmid vector pGX-attP [23] was kindly provided by Sergei A. Demakov (IMCB SB RAS, Novosibirsk, Russia).

To make a rescue construct pGE-attB-GMR-dCNDP2, we cloned a 4.3-kb genomic DNA fragment carrying the $d C N D P 2$ gene (2R:5,696,807-5,701,121) into the pGE-attB-GMR plasmid vector [23] using the unique NheI and AscI sites. The cloned DNA fragment contains the following eight single nucleotide variations within and nearby the $d C N D P 2$ gene: $5,697,584 \mathrm{C}>\mathrm{T}$ (upstream of the distal transcription start site), 5,698,421 T > A, $5,699,251 \mathrm{~T}>\mathrm{G}, 5,699,713 \mathrm{G}>\mathrm{T}, 5,699,725 \mathrm{del}$ (in the intronic regions), 5,699,463G $>\mathrm{A} \quad$ (synonymous 
substitution), 5,700,144 $\mathrm{T}>\mathrm{A}$ (in the $3^{\prime}$ untranslated region) and 5,700,278 $\mathrm{T}>\mathrm{C}$ (downstream of the transcription termination site). The plasmid vector pGE-attB-GMR [23] was kindly provided by Sergei A. Demakov (IMCB SB RAS, Novosibirsk, Russia).

To generate a pUASTattB-dCNDP2 construct for ectopic expression of the dCNDP2 protein, we first PCR-amplified the DNA sequence (corresponding to nucleotides 1001536 of GenBank accession no. NM_136337.3, but with 1023G > A and 1154A > T nucleotide substitutions) encoding the longer isoform of dCNDP2 (478 amino acids; hereafter dCNDP2-A). As a template, we used a cDNA synthesized from total RNA isolated from 0 to $24 \mathrm{~h}$ embryos from the wild-type Canton-S strain. Next, the amplified DNA fragment was cloned into the pUASTattB plasmid vector [24] by using the unique EcoRI and XbaI sites.

To make pUASTattB-eGFP-dCNDP2 and pUASTattBdCNDP2-eGFP constructs for ectopic expression of Nand C-terminal eGFP-tagged dCNDP2 fusion proteins, we used the full-length $d C N D P 2-A$ coding sequence (see above). It was fused in-frame either downstream or upstream of the eGFP coding sequence using site-directed mutagenesis by overlap extension [25]. The DNA fragments were then cloned into the pUASTattB plasmid vector [24] using the unique EcoRI and XbaI sites and the EcoRI and KpnI sites, respectively.

To produce the pGEX-4 T-dCNDP2 construct, the full-length $d C N D P 2-A$ coding sequence (see above) was cloned in-frame into the pGEX-4 T-1 plasmid vector (GE Healthcare) downstream of the glutathione S-transferase (GST) coding sequence using the BamHI and XhoI sites.

All plasmid constructs were verified by DNA sequencing. Details of plasmid constructions are available upon request.

\section{Germline genome editing and transgenesis}

To generate a null allele of $d C N D P 2$, the pU6-5' dCNDP2-chiRNA and pU6-3'dCNDP2-chiRNA targeting constructs and the donor pGX-5' \& $3^{\prime}$-dCNDP2-null construct, all dissolved in water, were mixed to final concentrations of $125 \mathrm{ng} / \mu \mathrm{l}, 125 \mathrm{ng} / \mu \mathrm{l}$ and $500 \mathrm{ng} / \mu \mathrm{l}$, respectively. The mixture was injected into embryos of the $w^{1118} ;$ PBac $\left\{y^{+m D i n t 2}=\right.$ vas-Cas9,U6-tracrRNA\}VK00027 strain (Bloomington stock \#51325) according to the standard procedure as previously described [26]. In total, 986 embryos were injected, out of which only 191 (19.4\%) developed to the adult stage. Transformants were identified in the $G_{1}$ progeny of fertile injectees by $w^{+}$ phenotype. We found only one such fly male, indicating that the efficiency of the CRISPR/Cas9-mediated HR at the $d C N D P 2$ locus was $0.1 \% \quad(1 / 986)$. The pGE-attB-GMR-dCNDP2 plasmid was injected at the concentration of $300 \mathrm{ng} / \mu \mathrm{l}$ into $\triangle d C N D P 2$ embryos expressing the phiC31 integrase in the germline [24]. The pUASTattB-dCNDP2, pUASTattB-eGFP-dCNDP2 and pUASTattB-dCNDP2-eGFP plasmids were individually injected at the concentration of $300 \mathrm{ng} / \mu \mathrm{l}$ into embryos carrying attP154 landing site [20] and expressing the phiC31 integrase in the germline [24].

\section{Genomic DNA extraction and PCR genotyping of engineered $d C N D P 2$ alleles}

To isolate genomic DNA, 3-5 flies were grinded in $200 \mu \mathrm{l}$ of DNA extraction buffer $(100 \mathrm{mM}$ Tris- $\mathrm{HCl}[\mathrm{pH}$ 7.5], $100 \mathrm{mM} \mathrm{NaCl}, 0.5 \%$ SDS, $50 \mathrm{mM}$ EDTA [pH 8.0], $200 \mathrm{mM}$ sucrose) in an $1.5-\mathrm{ml}$ tube and the sample was incubated at $65^{\circ} \mathrm{C}$ for $30 \mathrm{~min}$. Next, $300 \mu \mathrm{l}$ of $5 \mathrm{M} \mathrm{KAc}$ were added, mixed well by inversion and the sample was kept on ice for $30 \mathrm{~min}$. The tube was centrifuged for 15 min at $14,000 \mathrm{rpm}$. Then, the supernatant was transferred into a new tube and the DNA was precipitated with ethanol. Finally, the pellet was dissolved in 10$50 \mu \mathrm{l}$ of nuclease-free water. PCR was performed using Hot-Start Taq DNA polymerase (Biolabmix, MH010) according to the manufacturer's recommendations. For genotyping the $d C N D P 2$ locus after each step of its modification, allele-specific primer pairs were used (Additional file 1: Table S1). The PCR products were analyzed on $1 \%$ agarose gel along with GeneRuler $1 \mathrm{~Kb}$ Plus DNA Ladder (Thermo Scientific, SM1331).

\section{Anti-dCNDP2 antibody production}

The GST-dCNDP2 fusion protein was expressed in Escherichia coli strain BL21(DE3)pLysS (Promega) and subsequently purified as described previously [27]. The purified GST-dCNDP2 fusion protein was used to immunize mice. Polyclonal antibodies were affinity purified from serum as reported earlier [27].

\section{S2 cell culture and RNA interference (RNAi)}

First, we selected an 826-bp $d C N D P 2$ gene fragment, which is present in both transcript isoforms of the gene, as a template for the synthesis of double-stranded RNA (dsRNA). We amplified the DNA fragment using primers 5'-TAATACGACTCACTATAGGGAGGcgagatc ggtcg-3' and 5'-TAATACGACTCACTATAGGGAGGa tagcgccacctgg- $3^{\prime}$ that contain the $\mathrm{T} 7$ polymerase promoter sequence at their $5^{\prime}$ ends (shown in capital letters). The PCR product was purified using the GeneJET PCR Purification Kit (Thermo Scientific, K0702) and then used as a template to synthesize dsRNA as described earlier [28], with minor modifications. Treatment with DNaseI was done after heating the synthesized dsRNA to $65^{\circ} \mathrm{C}$ and its slow cooling to room temperature; in addition, the phenol/chloroform extraction was omitted. 
S2 cells were free from mycoplasma contamination and were cultured in $39.4 \mathrm{~g} / \mathrm{L}$ Shields and Sang M3 Insect medium (Sigma, S8398) supplemented with $0.5 \mathrm{~g} / \mathrm{L}$ $\mathrm{KHCO}_{3}$ and $20 \%$ heat-inactivated fetal bovine serum (FBS; Thermo Scientific, 10270106) at $25^{\circ} \mathrm{C}$. RNAi treatments were carried out as described previously [29], with the following modifications. Twenty-five $\mu \mathrm{g}$ of purified dsRNA was added to the cells three times (on the first, the third and the fifth days of incubation) and cells were harvested for analyses after 7 days of RNAi. Control S2 cell samples were prepared in the same way, but without addition of dsRNA.

\section{Western blotting}

Subcellular fractionation of normal S2 cells was conducted as described previously [30]. RNAi-treated and control S2 cells were harvested by centrifugation at 200 $g$ for $5 \mathrm{~min}$ at room temperature, washed with phosphate-buffered saline (PBS) and centrifuged again. S2 cell pellets and Drosophila embryos were homogenized and lysed in RIPA buffer (Sigma, R0278) containing $1 \times$ Halt $^{\mathrm{TM}}$ Protease and Phosphatase Inhibitor Cocktail (Thermo Scientific, 1861282). The lysates were clarified by centrifugation at $15,000 \mathrm{~g}$ for $15 \mathrm{~min}$ at $4{ }^{\circ} \mathrm{C}$ and the protein extracts were normalized using the DC Protein Assay (Bio-Rad, 5000116). Each normalized sample was mixed with an equal volume of $2 \times$ Laemmli buffer and incubated for $5 \mathrm{~min}$ at $95^{\circ} \mathrm{C}$ prior to analysis by SDS-PAGE and subsequent immunoblotting. Dissected larval and fly tissues were homogenized in $1 \times$ Laemmli buffer and incubated at $95^{\circ} \mathrm{C}$ for $5 \mathrm{~min}$ prior to analysis by SDS-PAGE and subsequent immunoblotting. The primary antibodies were mouse $\alpha$-Lamin Dm0 (1:300; Developmental Studies Hybridoma Bank, ADL67.10), mouse $\alpha$-alpha-tubulin (1:5000; Sigma, T6199), mouse $\alpha$-dCNDP2 antibody (1:10,000; this study), rabbit $\alpha$-Histone H3 (1:1000; Pierce, PA5-17697) and rabbit $\alpha$-GFP (1:2000; Sigma, G1544); they were detected using HRP-conjugated goat $\alpha$-mouse IgG (1:3500; Life Technology, G-21040) and goat $\alpha$-rabbit IgG (1:3500; Life Technology, G-21234). Images were captured using an Amersham Imager 600 System (GE Healthcare).

\section{Immunofluorescence (IF) staining}

Immunostaining of S2 cells and squashed salivary gland polytene chromosomes was performed as described previously [31, 32]. The primary mouse polyclonal $\alpha$-dCNDP2 antibodies were used at dilution 1:1000 and detected by goat $\alpha$-mouse IgG antibodies conjugated to Alexa Fluor 488 (1:500; Invitrogen, A-11001). IF images of S2 cells were made using Zeiss LSM 710 confocal microscope and an oil $100 \times / 1.40$ plan apo lens. IF images of polytene chromosomes were acquired with a Zeiss Axio Observer.Z1 fluorescence microscope equipped with an Axiocam 506 mono (D) camera using an oil $63 \times / 1.40$ plan apo lens. In both cases, ZEN 2012 software was used for image acquisition.

For detection of eGFP-tagged dCNDP2 fusion proteins, whole salivary glands were fixed in PBS containing 4\% formaldehyde (Merck, 104003), washed 3 times for $5 \mathrm{~min}$ each with PBS containing $0.5 \%$ Triton X-100, stained for $30 \mathrm{~min}$ with $0.4 \mu \mathrm{g} / \mathrm{ml}$ DAPI dissolved in PBS and mounted in 50\% Glycerol dissolved in PBS. IF images of whole-mounted glands were obtained on a Zeiss LSM 710 confocal microscope using a 20×/0.50 EC Plan-Neofluar lens. Optical sections were combined using the LSM Image Browser version 3.5 software (Zeiss).

\section{Results}

To address the function of $d C N D P 2$, we generated a null mutation in the gene, $\triangle d C N D P 2$, using the recently developed CRISPR/Cas9-mediated HR method [21], which is illustrated in detail in Fig. 1 and in Additional file 2: Figure S1. The final product of this procedure was the $\triangle d C N D P 2$ null mutation that carries a phiC31 attP site instead of the $\sim 4.3-\mathrm{kb}$ DNA sequence containing the entire $d C N D P 2$ gene. We found that homozygous mutants do not display visible morphological abnormalities and are fertile. We also used phiC31 integrase-mediated recombination to deliver the same $\sim 4.3-\mathrm{kb}$ DNA fragment that was deleted in $\triangle d C N D P 2$ back into its original genomic location, and generated the $\triangle d C N D P 2^{\text {(rescue) }}$ allele (Fig. 1; Additional file 2: Figure S1).

The wild-type $d C N D P 2$ gene is predicted to encode two protein isoforms that are slightly different in their amino acid sequences (the $\mathrm{A}$ and $\mathrm{C}$ isoforms, with molecular weights of $53.2 \mathrm{kDa}$ and $47.9 \mathrm{kDa}$, respectively; see flyBase [15]). Namely, the shorter isoform does not contain the first 47 amino acids that are present at the $\mathrm{N}$-terminus of the longer isoform. To analyze the expression pattern of dCNDP2 protein isoforms during Drosophila development, we generated antibodies using the full-length dCNDP2-A isoform as an antigen. The specificity of the antibodies was first assessed by Western blotting of protein extracts prepared from normal S2 cells and S2 cells treated with $d C N D P 2$ dsRNA. In control cells, we observed a clear signal of the expected molecular weight that was almost absent in RNAi cells (Fig. 2a). Western blotting with $\alpha$-dCNDP2 antibodies showed the absence of the protein also in embryos, ovaries, brains and salivary glands of $\triangle d C N D P 2$ mutants (Fig. 2b-e), and the expected restoration of dCNDP2 expression level in $\triangle d C N D P 2^{\text {(rescue) }}$ flies (Fig. $2 \mathrm{~d}$, e). Interestingly, in most tissues examined, the antibodies recognized a single band, presumably corresponding to the dCNDP2-A isoform (Fig. 2b-e). Only in 4-6h old embryos we detected a second faint band that might correspond to the smaller predicted isoform of 


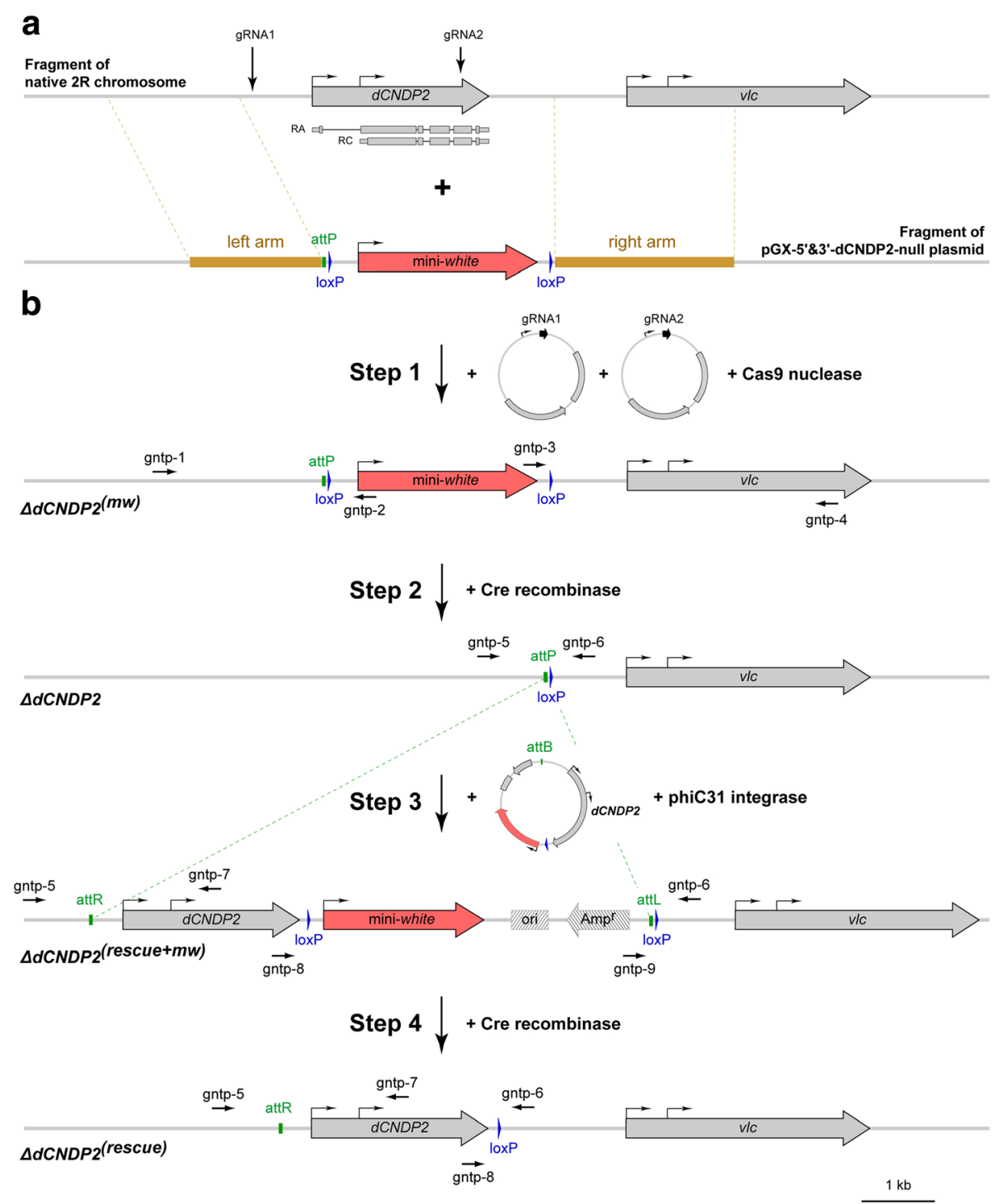

Fig. 1 Experimental design for generation of null and rescue alleles of the dCNDP2 gene. a Schematic representation of the dCNDP2 locus and the donor plasmid pGX-5'\&3'-dCNDP2-null, which contains the mini-white reporter gene (red) flanked by the left and right homology arms (brown). The gRNA target sites are indicated by vertical arrows. b Modifications introduced into the dCNDP2 locus. In step 1, the dCNDP2 gene was replaced by the mini-white reporter gene using CRISPR/Cas9-mediated HR approach. Note that the reporter gene is flanked by two loxP sites. In step 2, the mini-white reporter was removed by Cre recombinase-mediated recombination between loxP sites. This resulted in generation of a null allele ( $\triangle d C N D P 2)$, in which the $d C N D P 2$ gene is replaced by the attP and loxP sites. In step 3, the rescue construct pGE-attB-GMR-dCNDP2 was integrated into the attP site by phiC31 integrase-mediated recombination. The rescue construct contains the mini-white reporter gene, which upon integration into the genome becomes flanked by two loxP sites, and the genomic DNA fragment carrying the dCNDP2 gene that is absent in $\triangle d C N D P 2$ mutants. In step 4, the mini-white reporter was removed by Cre recombinase-mediated recombination between loxP sites. This resulted in generation of the $\triangle d C N D P 2^{\text {(rescue) }}$ transgenic flies, in which DNA sequence of the dCNDP2 locus is almost identical to the wild-type one except for several single nucleotide variations (see Methods) and the presence of the attR and loxP sites. Black horizontal arrows indicate primers used for PCR genotyping (for primers sequences, see Additional file 1: Table S1). Plasmids are drawn as circles with the relevant elements indicated; Ampr', ampicillin resistance gene; ori, plasmid origin of replication

dCNDP2 (Fig. 2b). These results suggest that only one of the two predicted isoforms of the dCNDP2 protein is expressed in most tissues.

To determine the subcellular localization of dCNDP2, we first immunostained S2 cells with $\alpha$-dCNDP2 antibodies. We found that the protein is present in both the nucleus and the cytoplasm, and that the nuclei exhibit an unstained region that could be the nucleolus (Fig. 2f). Consistent with Western blotting analysis, both the nuclear and cytoplasmic stainings were 


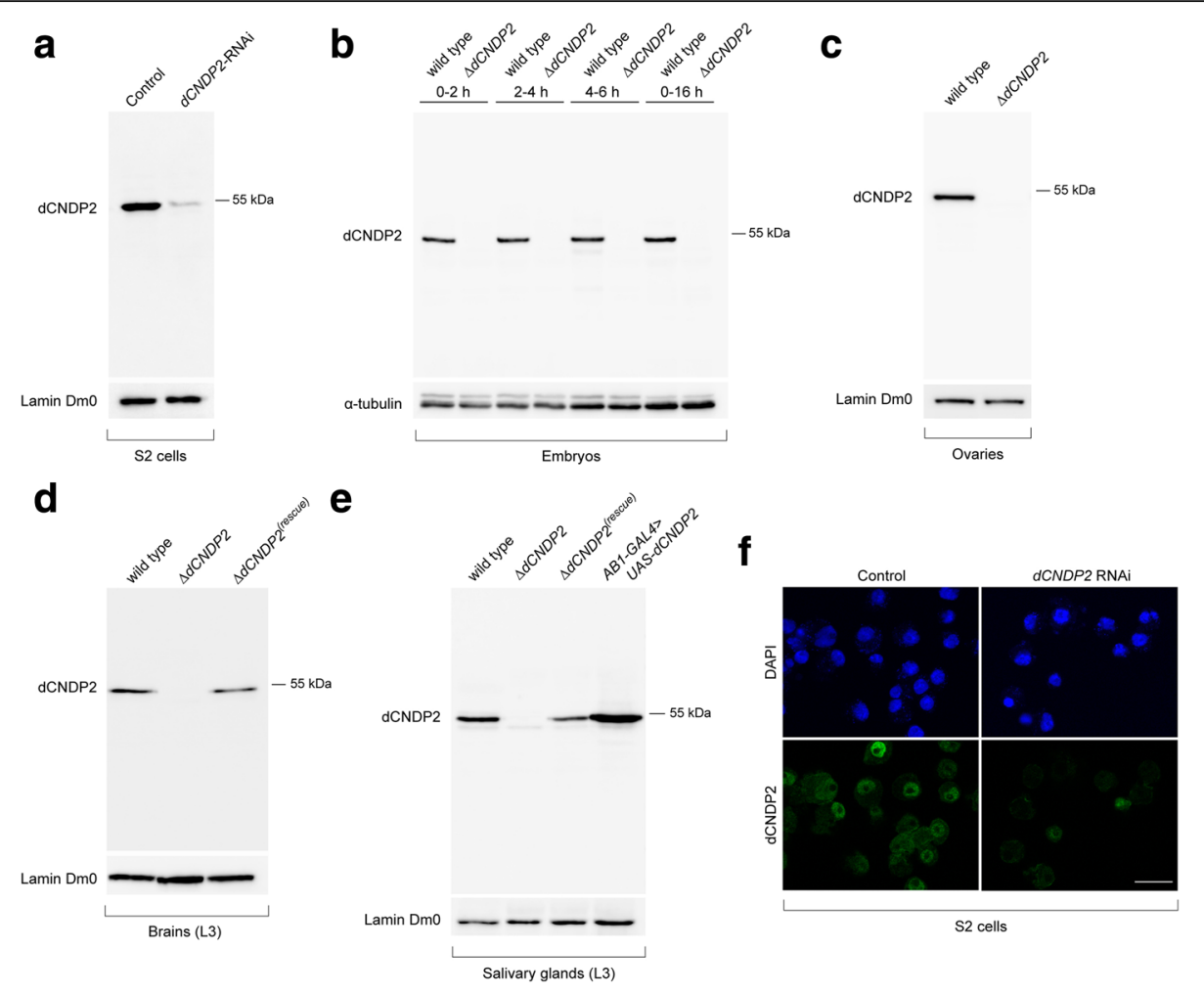

Fig. 2 a-dCNDP2 antibodies are specific to the target protein. a-e Western blots showing that the a-dCNDP2 antibodies recognize a single band migrating slightly lower than the $55 \mathrm{kDa}$ standard. This band is likely to correspond to the $53.2 \mathrm{kDa}$ isoform A. a S2 cells; b embryos; c ovaries; d larval brains; e salivary glands. L3, third instar larval stage. Note that in 4-6 h old embryos the antibodies recognize a second faint band that might correspond to the $47.9 \mathrm{kDa}$ isoform C. f Immunostaining of dCNDP2 depleted (dCNDP2 RNAi) and control S2 cells with a-dCNDP2 antibodies. Scale bar, $20 \mu \mathrm{m}$

dramatically reduced after the depletion of the protein by RNAi (Fig. 2f). The localization pattern of dCNDP2 was confirmed by subcellular fractionation of S2 cell extracts. This analysis revealed that the protein is predominantly cytoplasmic, but it is also a soluble nuclear component (Fig. 3a).

We also generated transgenic fly lines expressing $\mathrm{N}$ - or C-terminally eGFP-tagged dCNDP2 under the control of the UAS promoter. When eGFP-dCNDP2 and dCNDP2eGFP proteins were expressed using AB1-GAL4 driver, both recombinant proteins were predominantly localized within the nucleus of salivary gland cells of third instar larvae (Fig. 3b, c). We then immunostained salivary gland polytene chromosomes from $\triangle d C N D P 2$, $\triangle d C N D P 2^{\text {(rescue) }}$ and $A B 1-G A L 4>U A S$-dCNDP2 larvae with $\alpha$-dCNDP2 antibodies and found that dCNDP2 associates with multiple chromosomal sites (Fig. 3d). These results indicate that the Drosophila dCNDP2 protein, as its mammalian counterpart, is a component of both the cytoplasm and the nucleus. In addition, $\mathrm{dCNDP} 2$ protein is able to bind the chromatin of polytene chromosomes.

\section{Discussion}

Our knowledge on consequences of the complete loss of the CNDP2 gene function in humans is very limited. There is only a single case report of a likely complete deficiency of this gene, which is associated with global developmental delays, ataxia, hypotonia and tremor [33]. Yet, in this case, the presence of a gross chromosomal deletion does not allow a firm conclusion on whether the effects are caused solely by CNDP2 deficiency [33]. Thus, it is difficult to decide whether the phenotypic consequences of Drosophila $d C N D P 2$ loss are substantially different from those elicited by the loss of the human homolog.

Many studies reported that the CNDP2 gene is misexpressed in different types of human cancers [2, 611]. CNDP2 was also found to be involved in the regulation of the cell cycle in human cancer cell lines. Specifically, CNDP2 overexpression in pancreatic cancer lines induced the accumulation of cells in the G0/ G1 phase [6], whereas knockdown of CNDP2 in colon cancer cells blocked the cell cycle progression in the G2/M phase [11]. However, the molecular mechanisms underlying these effects have not been 

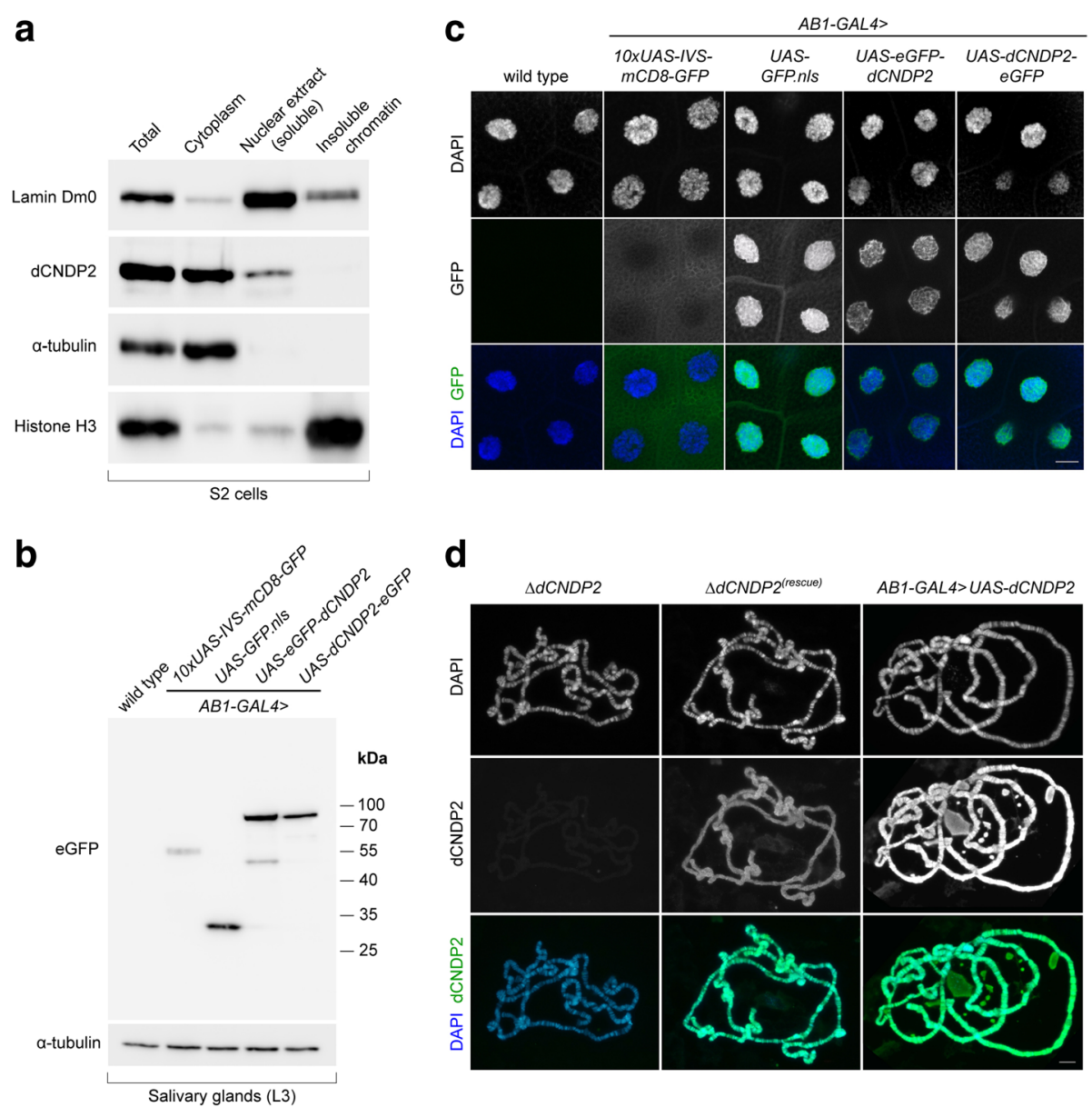

Fig. 3 dCNDP2 is present in both the cytoplasm and the nucleus. a Western blot showing subcellular fractionation of S2 cells. b Western blot showing ectopic expression of eGFP-tagged proteins in salivary glands using the AB1-GAL4 driver. L3, third instar larval stage. $\mathbf{c}$ Localization of eGFP-tagged proteins in L3 salivary gland cells. GFP.nls and mCD8:GFP were used as controls for nuclear localization, and cytoplasmic and transmembrane localization, respectively. Scale bar, $20 \mu \mathrm{m}$. $\mathbf{d}$ dCNDP2 binds multiple sites on polytene chromosomes. The following exposure

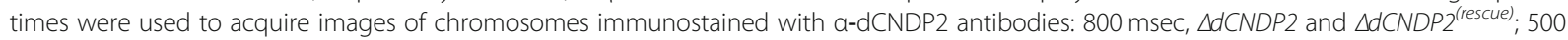
msec, AB1-GAL4 > UAS-dCNDP2. Scale bar, $20 \mu \mathrm{m}$

uncovered. Thus, it will be interesting investigating whether dCNDP2 is implicated in the control of the Drosophila cell cycle.

The binding of dCNDP2 to polytene chromosomes suggests that it may be involved in the regulation of processes such as DNA replication, transcription or repair. However, we have limited information on how this protein is transported into the nucleus. Typically, the nuclear localization of a protein larger than $40-45 \mathrm{kDa}$ is controlled by the presence of a nuclear localization signal (NLS) of a specific amino acid sequence (for review, see [34-36]). Indeed, we predicted an importin- $\alpha / \beta$ pathway-specific monopartite NLS (YWLGKKRPCLTY) in the middle portion of the dCNDP2 protein, using the cNLS Mapper (nls-mapper.iab.keio.ac.jp/cgi-bin/NLS_Map per_form.cgi [37]). However, the functionality of this motif as a NLS has not been tested so far. A definition of the dCNDP2 NLS could help designing experiments aimed at understanding of the role of this protein in the nucleus.

\section{Conclusions}

We developed a set of genetic and molecular tools to study the function of the dCNDP2 protein in D. melanogaster. Particularly, we generated the $d C N D P 2$ null allele using a CRISPR/Cas9-mediated HR approach and different transgenic flies to overexpress $d C N D P 2$. We demonstrated that this gene is not essential for fly viability under standard laboratory conditions. Although two $\mathrm{dCNDP} 2$ isoforms with different molecular weights have been previously predicted, we detected the presence of only one of them in most Drosophila tissues. Moreover, we found that dCNDP2 is localized not only in the 
cytoplasm, but also in the nucleus, where it can be associated with chromatin. Thus, both the amino acid sequence of the protein and its subcellular localization are conserved between mammals and fruit flies. This indicates that $D$. melanogaster is a good model for further elucidation of the mechanisms of CNDP2 action in the context of the whole organism.

\section{Additional files}

Additional file 1: Table S1. Primers used for PCR genotyping of dCNDP2 alleles and PCR product details. (PDF $139 \mathrm{~kb}$ )

Additional file 2: Figure S1. Molecular verification of $d C N D P 2$ alleles. (PDF $553 \mathrm{~kb}$ )

\section{Abbreviations}

$\triangle d C N D P 2:$ dCNDP2 null mutation; Cas9: CRISPR associated protein 9: chiRNA: Chimeric RNA; CHO cells: Chinese hamster ovary cells; CN2: Carnosine dipeptidase II; CNDP2: Cytosolic non-specific dipeptidase 2;

CPGL: Carboxypeptidase of glutamate-like; CRISPR: Clustered regularly interspaced short palindromic repeats; DAPI: 4',6-diamidino-2-phenylindole; dCNDP2: Drosophila CNDP2; dsRNA: Double-stranded RNA; eGFP: Enhanced green fluorescent protein; FBS: Fetal bovine serum; GMR: Glass multiple reporter; gRNA: Guide RNA; HR: Homologous recombination; HRP: Horseradish peroxidase; IF: Immunofluorescence; NLS: Nuclear localization signal; PBS: Phosphate-buffered saline; RNAi: RNA interference; SDS-PAGE: Sodium dodecyl sulphate-polyacrylamide gel electrophoresis; tracrRNA: Trans-activating crRNA; UAS: Upstream activation sequence

\section{Acknowledgments}

We thank Julia V. Popova for technical assistance with experiments; Melissa Harrison, Kate O'Connor-Giles, Jill Wildonger, Sergei A. Demakov, Stepan N. Belyakin for providing plasmid vectors and fly stocks for CRISPR/Cas9- and phiC31-mediated transgenesis; and Maurizio Gatti for critical reading of the manuscript. The microscopy and DNA sequencing were performed using resources provided by the Molecular and Cellular Biology core facility of the Institute of Molecular and Cellular Biology of the Siberian Branch of the Russian Academy of Sciences. Antibodies were raised at the Center for Genetic Resources of Laboratory Animals, Institute of Cytology and Genetics, Siberian Branch of the Russian Academy of Sciences (RFMEFI61914X0005 and RFMEFI61914X0010)

\section{Funding}

This work was supported by a grant from the Russian Foundation for Basic Research (16-04-01598), by a Russian Science Foundation grant (16-14-10288) for ectopic expression of eGFP-tagged proteins, and by a project of the Fundamental Scientific Research Program of the Siberian Branch of the Russian Academy of Sciences (0310-2019-0005) for generation of antibodies specific to dCNDP2. Publication costs are funded by a grant from the Russian Foundation for Basic Research (16-04-01598).

\section{Availability of data and materials}

All supporting data are included in the additional files. The materials generated during the current study are available from the corresponding authors on reasonable request.

\section{About this supplement}

This article has been published as part of BMC Genetics Volume 20 Supplement 1 , 2019: Selected articles from BGRSISB-2018: genetics. The full contents of the supplement are available online at https://bmcgenet.biomedcentral.com/ articles/supplements/volume-20-supplement-1.

\section{Authors' contributions}

Conceived and designed the experiments: AVP, ENA. Performed the experiments: ENA, AAO, TDD, ALO, ENK, AVI, GAP, SAK. Analyzed the data: ENA, AAO, AVP. Wrote the paper: ENA, AAO and AVP. All authors read and approved the final version of the manuscript.
Ethics approval and consent to participate

Not applicable

\section{Consent for publication}

Not applicable

\section{Competing interests}

The authors declare that they have no competing interests.

\section{Publisher's Note}

Springer Nature remains neutral with regard to jurisdictional claims in published maps and institutional affiliations.

\section{Author details}

${ }^{1}$ Institute of Molecular and Cellular Biology, Siberian Branch of the Russian Academy of Sciences, Novosibirsk 630090, Russia. ${ }^{2}$ Novosibirsk State University, Novosibirsk 630090, Russia. ${ }^{3}$ Institute of Chemical Biology and Fundamental Medicine, Siberian Branch of the Russian Academy of Sciences, Novosibirsk 630090, Russia.

Published: 18 March 2019

References

1. Teufel M, Saudek V, Ledig JP, Bernhardt A, Boularand S, Carreau A, Cairns NJ, Carter C, Cowley DJ, Duverger D, et al. Sequence identification and characterization of human carnosinase and a closely related non-specific dipeptidase. J Biol Chem. 2003;278(8):6521-31.

2. Zhang P, Chan DW, Zhu YY, Li JJ, Ng IOL, Wan D, Gu J. Identification of carboxypeptidase of glutamate like- $B$ as a candidate suppressor in cell growth and metastasis in human hepatocellular carcinoma. Clin Cancer Res. 2006;12(22):6617-25

3. Okumura N, Tamura J, Takao T. Evidence for an essential role of intradimer interaction in catalytic function of carnosine dipeptidase II using electrospray-ionization mass spectrometry. Protein Sci. 2016;25(2):511-22.

4. Okumura N, Takao T. The zinc form of carnosine dipeptidase 2 (CN2) has dipeptidase activity but its substrate specificity is different from that of the manganese form. Biochem Biophys Res Commun. 2017;494(3-4):484-90.

5. Jansen RS, Addie R, Merkx R, Fish A, Mahakena S, Bleijerveld OB, Altelaar M, L IJlst, Wanders RJ, Borst $\mathrm{P}$, et al. N-lactoyl-amino acids are ubiquitous metabolites that originate from CNDP2-mediated reverse proteolysis of lactate and amino acids. Proc Natl Acad Sci USA. 2015;112(21):6601-6.

6. Lee JH, Giovannetti E, Hwang JH, Petrini I, Wang Q, Voortman J, Wang Y, Steinberg SM, Funel N, Meltzer PS, et al. Loss of 18q22.3 involving the carboxypeptidase of glutamate-like gene is associated with poor prognosis in resected pancreatic cancer. Clin Cancer Res. 2012;18(2):524-33.

7. Zhang Z, Miao L, Xin X, Zhang J, Yang S, Miao M, Kong X, Jiao B. Underexpressed CNDP2 participates in gastric cancer growth inhibition through activating the MAPK signaling pathway. Mol Med. 2014;20:17-28.

8. Tripathi A, King C, de la Morenas A, Perry VK, Burke B, Antoine GA, Hirsch EF, Kavanah M, Mendez J, Stone M, et al. Gene expression abnormalities in histologically normal breast epithelium of breast cancer patients. Int J Cancer. 2008:122(7):1557-66.

9. Perroud B, Ishimaru T, Borowsky AD, Weiss RH. Grade-dependent proteomics characterization of kidney cancer. Mol Cell Proteomics. 2009:8(5): 971-85

10. Okamura N, Masuda T, Gotoh A, Shirakawa T, Terao S, Kaneko N, Suganuma K, Watanabe M, Matsubara T, Seto R, et al. Quantitative proteomic analysis to discover potential diagnostic markers and therapeutic targets in human renal cell carcinoma. Proteomics. 2008;8(15):3194-203.

11. Xue C, Zhang Z, Yu H, Yu M, Yuan K, Yang T, Miao M, Shi H. Up-regulation of CNDP2 facilitates the proliferation of colon cancer. BMC Gastroenterol. 2014;14:96.

12. Eccleston ED, Gerenday A, Fallon AM. Leveraging genomic databases: from an Aedes albopictus mosquito cell line to the malaria vector Anopheles gambiae via the Drosophila genome project. Insect Mol Biol. 2002;11(2): 187-95.

13. Kaur H, Kumar C, Junot C, Toledano MB, Bachhawat AK. Dug1p is a Cys-Gly peptidase of the gamma-glutamyl cycle of Saccharomyces cerevisiae and represents a novel family of Cys-Gly peptidases. J Biol Chem. 2009;284(21): $14493-502$. 
14. Myster SH, Wang F, Cavallo R, Christian W, Bhotika S, Anderson CT, Peifer M. Genetic and bioinformatic analysis of $41 \mathrm{C}$ and the $2 \mathrm{R}$ heterochromatin of Drosophila melanogaster: a window on the heterochromatin-euchromatin junction. Genetics. 2004;166(2):807-22.

15. Gramates LS, Marygold SJ, Santos GD, Urbano JM, Antonazzo G, Matthews BB, Rey AJ, Tabone CJ, Crosby MA, Emmert DB, et al. FlyBase at 25: looking to the future. Nucleic Acids Res. 2017:45(D1):D663-71.

16. Uhlen M, Zhang C, Lee S, Sjöstedt E, Fagerberg L, Bidkhori G, Benfeitas R, Arif M, Liu Z, Edfors F, et al. A pathology atlas of the human cancer transcriptome. Science. 2017;357(6352):eaan2507.

17. Vierstraete E, Cerstiaens A, Baggerman G, Van den Bergh G, De Loof A, Schoofs L. Proteomics in Drosophila melanogaster: first 2D database of larval hemolymph proteins. Biochem Biophys Res Commun. 2003;304(4):831-8.

18. Karlsson C, Korayem AM, Scherfer C, Loseva O, Dushay MS, Theopold U. Proteomic analysis of the Drosophila larval hemolymph clot. J Biol Chem. 2004;279(50):52033-41.

19. Laktionov PP, White-Cooper H, Maksimov DA, Belyakin SN. Transcription factor Comr acts as a direct activator in the genetic program controlling spermatogenesis in D. melanogaster. Molecular Biology (Moscow). 2014; 48(1):130-40.

20. Markstein M, Pitsouli C, Villalta C, Celniker SE, Perrimon N. Exploiting position effects and the gypsy retrovirus insulator to engineer precisely expressed transgenes. Nat Genet. 2008;40(4):476-83.

21. Gratz SJ, Cummings AM, Nguyen JN, Hamm DC, Donohue LK, Harrison MM, Wildonger J, O'Connor-Giles KM. Genome engineering of Drosophila with the CRISPR RNA-guided Cas9 nuclease. Genetics. 2013; 194(4):1029-35

22. Hoskins RA, Carlson JW, Wan KH, Park S, Mendez I, Galle SE, Booth BW, Pfeiffer BD, George RA, Svirskas R, et al. The release 6 reference sequence of the Drosophila melanogaster genome. Genome Res. 2015; 25(3):445-58.

23. Huang J, Zhou W, Dong W, Watson AM, Hong Y. Directed, efficient, and versatile modifications of the Drosophila genome by genomic engineering. Proc Natl Acad Sci USA. 2009;106(20):8284-9.

24. Bischof J, Maeda RK, Hediger M, Karch F, Basler K. An optimized transgenesis system for Drosophila using germ-line-specific phiC31 integrases. Proc Natl Acad Sci U S A. 2007;104(9):3312-7.

25. Ho SN, Hunt HD, Horton RM, Pullen JK, Pease LR. Site-directed mutagenesis by overlap extension using the polymerase chain reaction. Gene. 1989;77(1):51-9.

26. Ringrose L. Transgenesis in Drosophila melanogaster. Methods Mol Biol. 2009;561:3-19.

27. Chalkley GE, Verrijzer CP. Immuno-depletion and purification strategies to study chromatin-remodeling factors in vitro. Methods Enzymol. 2004;377:421-42.

28. Somma MP, Fasulo B, Cenci G, Cundari E, Gatti M. Molecular dissection of cytokinesis by RNA interference in Drosophila cultured cells. Mol Biol Cell. 2002;13(7):2448-60.

29. Somma MP, Ceprani F, Bucciarelli E, Naim V, De Arcangelis V, Piergentili R, Palena A, Ciapponi L, Giansanti MG, Pellacani C, et al. Identification of Drosophila mitotic genes by combining co-expression analysis and RNA interference. PLoS Genet. 2008;4(7):e1000126.

30. Rodrigue A, Lafrance M, Gauthier MC, McDonald D, Hendzel M, West SC Jasin M, Masson JY. Interplay between human DNA repair proteins at a unique double-strand break in vivo. EMBO J. 2006;25(1):222-31.

31. Strunov A, Boldyreva LV, Andreyeva EN, Pavlova GA, Popova JV, Razuvaeva AV, Anders AF, Renda F, Pindyurin AV, Gatti M, et al. Ultrastructural analysis of mitotic Drosophila S2 cells identifies distinctive microtubule and intracellular membrane behaviors. BMC Biol. 2018;16(1):68.

32. Andreyeva EN, Bernardo TJ, Kolesnikova TD, Lu X, Yarinich LA, Bartholdy BA, Guo X, Posukh OV, Healton S, Willcockson MA, et al. Regulatory functions and chromatin loading dynamics of linker histone $\mathrm{H} 1$ during endoreplication in Drosophila. Genes Dev. 2017;31(6):603-16.

33. Willi SM, Zhang Y, Hill JB, Phelan MC, Michaelis RC, Holden KR. A deletion in the long arm of chromosome 18 in a child with serum carnosinase deficiency. Pediatr Res. 1997;41(2):210-3.

34. Jans DA. The regulation of protein transport to the nucleus by phosphorylation. Biochem J. 1995;311(Pt 3):705-16.

35. Bauer NC, Doetsch PW, Corbett $\mathrm{AH}$. Mechanisms regulating protein localization. Traffic. 2015;16(10):1039-61.
36. Christie M, Chang CW, Róna G, Smith KM, Stewart AG, Takeda AAS, Fontes MRM, Stewart M, Vértessy BG, Forwood JK, et al. Structural biology and regulation of protein import into the nucleus. J Mol Biol. 2016;428(10 Pt A): 2060-90.

37. Kosugi S, Hasebe M, Tomita M, Yanagawa H. Systematic identification of cell cycle-dependent yeast nucleocytoplasmic shuttling proteins by prediction of composite motifs. Proc Natl Acad Sci U S A. 2009;106(25):10171-6.
Ready to submit your research? Choose BMC and benefit from:

- fast, convenient online submission

- thorough peer review by experienced researchers in your field

- rapid publication on acceptance

- support for research data, including large and complex data types

- gold Open Access which fosters wider collaboration and increased citations

- maximum visibility for your research: over $100 \mathrm{M}$ website views per year

At BMC, research is always in progress.

Learn more biomedcentral.com/submissions 